\title{
Development of novel mobile robot with semicircular wheels
}

\author{
Kanta Yamamoto *i[ and Takeshi Aoki
}

\begin{abstract}
This paper describes development of a mobile robot using semicircular wheels on uneven terrain. It performs the crawl gait on flat ground without changing potential energy and the butterfly gait to climb over debris. We also used crawl gait to perform turning motion. The butterfly gait is used to climb over high steps. Turning motion is achieved not only by creating a difference in angular velocity between the wheels on both sides, but also by keeping the crawl gait. We achieved continuous movement from turning motion to pivot turning. We conducted an experiment on the movement and turning motion on flat ground using crawl gait.
\end{abstract}

\section{Introduction}

Many mobile robots that travel on rough terrain have been developed and are expected to be active as rescue robots. We have developed a prototype model that can be used in buildings that might collapse. Rescue workers go into buildings to look for victims, often when there is risk of secondary disaster. If the building did not completely collapse and most floors remain flat, mobility using wheels is more efficient. Also, because there may be obstacles such as stairs and daily necessities, the ability to climb steps is required. Those based on the Tristar [1] comprise a large Tri-star shaped wheel and three small wheels arranged in an equilateral triangle. When a small wheel becomes immovable, the large wheel starts to rotate and function as one leg. The wheels of Tribot [2] and tri-wheel [3] were developed based on the Tri-star wheel. Two wheels are in contact with the ground. The three wheels are active wheels and move with two wheels on the ground to move along flat ground. Beyond that, three wheels are treated as legs and handle step climbing functions. Tri-wheel is a novel wheel developed by Lauren M. Smith. The three wheels are active wheels. There are two modes: Driving mode and Tumbling mode. The mode is changed when moving along flat ground and in step climbing. The prototype model that focused on

\footnotetext{
*Correspondence: s1526120qh@s.chibakoudai.jp
}

Chiba Institute of Technology, Narashino, Japan this movement method is Loper [4]. Loper is a quadruped-hybrid stair-climbing robot developed by Sam D. Herbert. It uses a new wheel called Tri-lobe. The wheel is based on the Tri-Star Wheel; it adopts a method that drives a leg. The Tribot and Tri-Star Wheels can move with high step climbing performance and static stability on flat ground. However, to achieve compatibility, wheel mechanisms become complicated. Although Loper wheels are simple, intense vertical shaking occurs when traveling on flat ground, generating unneeded energy. It also burdens the operator. RHex $[5,6]$ and Transleg $[7,8]$ were developed for leg-wheel type robots. RHex is a simple highly mobile hexapod robot $[5,6]$. The legs are semicircular, and because they are made of glass fibers, they have both elasticity and robustness. RHex uses the tripod gait as basic locomotion, making it possible to handle activities with big rocks and rough terrain. Transleg [7, 8] is a deformable leg wheel robot developed by Zhongn Wei. Deformable legs are stored on the side of the circular wheel and the legs are expanded using wires. Using the circular wheels and legs properly, Transleg will move along flat ground and will climb steps. A problem with these robots is that RHex has a short moving distance with respect to the diameter of its legs. Although the upward and downward motion on flat ground is intense, its moving efficiency is decreased. Transleg has a deformation mechanism between the circular wheels and legs. Also, the mechanism becomes complicated. Sojourner
Springer Open

(c) The Author(s) 2020. This article is licensed under a Creative Commons Attribution 4.0 International License, which permits use, sharing adaptation, distribution and reproduction in any medium or format, as long as you give appropriate credit to the original author(s) and the source, provide a link to the Creative Commons licence, and indicate if changes were made. The images or other third party material in this article are included in the article's Creative Commons licence, unless indicated otherwise in a credit line to the material. If material is not included in the article's Creative Commons licence and your intended use is not permitted by statutory regulation or exceeds the permitted use, you will need to obtain permission directly from the copyright holder. To view a copy of this licence, visit http://creativeco mmons.org/licenses/by/4.0/. 
[9] and Rocker-Pillar [10] have been developed as robots having a rocker bogie mechanism. Sojourner is a robot developed to work on Mars. Because it has a steering mechanism, it can be made to turn with no speed difference. Rocker-Pillar is a crawler robot and can move even on terrain that cannot be navigated on wheels. A problem is that the rocker bogie mechanism must comprise too many links, complicating its structure [11]. The main problem with many of the mobile robots discussed is their complicated climbing mechanism. We believe the mechanism must be simplified because complicated mechanisms result in mobile robots that are large and heavy. To solve this problem, we propose wheels of simple shape, such as a semicircular shape. A mobile robot having semicircular wheels cannot move smoothly without pitching because the wheels are not circular. To solve the problem, we installed the gait of legged robots to a wheel-type mobile robot. However, this gait causes falls when turning because the structure of wheel-type robots differs from that of leg robots. We therefore propose a new turning method.

The remainder of this paper is organized as follows; Section II describes the design concept of a semicircular wheel and presents a prototype model; Section III shows details of the prototype model we developed; Section IV describes the moving method; and Section V describes the turning motion.

\section{Design concept}

\section{Consideration of wheel shape}

As previously mentioned, a requirement for the novel wheel we developed is that it be able to move as well as a circular wheel without deterioration in step climbing performance compared to other special wheels, and when traveling on flat ground. Figure 1 shows performance comparisons of various wheel shapes that can climb a low step. The circle wheel has no convex portions on the circumference; the grouser wheel has them. However, the grouser wheel cannot hook an edge of a step higher than its radius of a wheel by a convex portion. On the other hand, a semicircular wheel can hook the edge of a step higher than its radius. These results revealed that the semicircular wheel has the highest step climbing performance.

\section{Basic concept of prototype model}

Figure 2 is the basic design of the prototype model to be developed. It is a four-wheeled model equipped with semicircular wheels. Each wheel has an actuator to control its angle and speed of wheels individually. By connecting the arcs of four wheels continuously, it is thereby possible to move without pitching upward and downward on flat ground.
Bari-Bari [12] was developed as a mobile robot that traverses rough terrain by moving the body and other parts alternately. It traverses well in rough terrain. As a similar movement, we focused on the butterfly gait. It can move by rotating all the wheels in the same phase; using legs and body, it produces large thrust force. Also, in an unstable place, as shown in Fig. 3, the ground pressure is dispersed by several contact points of the body with the ground. As a result, the prototype model is stable even in uneven places.

\section{Design of prototype model \\ Overview}

We developed a prototype model based on the basic design. Figure 4 is an overview of the prototype model and Table 1 lists it specifications. The semicircular wheel has an arc-shaped part and a linear part at 180 degree intervals. Using a 3D printer, I have maintained robustness while reducing weight using a lattice structure inside the wheel. Also, a non-slip seat is attached around the wheel to prevent slipping. Without the nonslip seat, the wheel slips on the ground. Also, as shown in Fig. 5, when a convex is attached to the tip of the wheel, this convexity is caught to assist in step climbing.

\section{Motion experiment of butterfly gait}

We conducted an experiment to confirm the step climbing ability of the prototype model. The experiment uses a step of wood $110 \mathrm{~mm}$ high. All wheels rotate in the same phase with rotation at $70 \mathrm{deg} / \mathrm{s}$. Figure 6, (1) to (6) indicates the process as the prototype model goes through a step. The experiment confirmed that the prototype model did not overturn and that it could climb a step of $110 \mathrm{~mm}$. To conduct a comparative experiment, we did the same experiment using grouser wheels of the same diameter. It could not climb the step (Fig. 7) because the protrusions of the grouser wheels could not hook the edge of the step. This result shows that semicircular wheels have a higher step climbing ability than grouser wheels.

With its current body length, the prototype model cannot climb more than $110 \mathrm{~mm}$. However, as shown in Fig. 8, the prototype model can reach the edge of a step $190 \mathrm{~mm}$ high by the front wheels. Extending its body length makes it possible to climb over this step height.

\section{Consideration of locomotion}

\section{Installation of crawl gait}

Crawl gait combines the actions of semicircular wheels and makes the robot travel in the same way as circular wheels. With circular wheels, a robot can move without special control. With semicircular wheels, however, a robot needs proper control because the noncircular shape cannot produce smooth movement of the robot. 

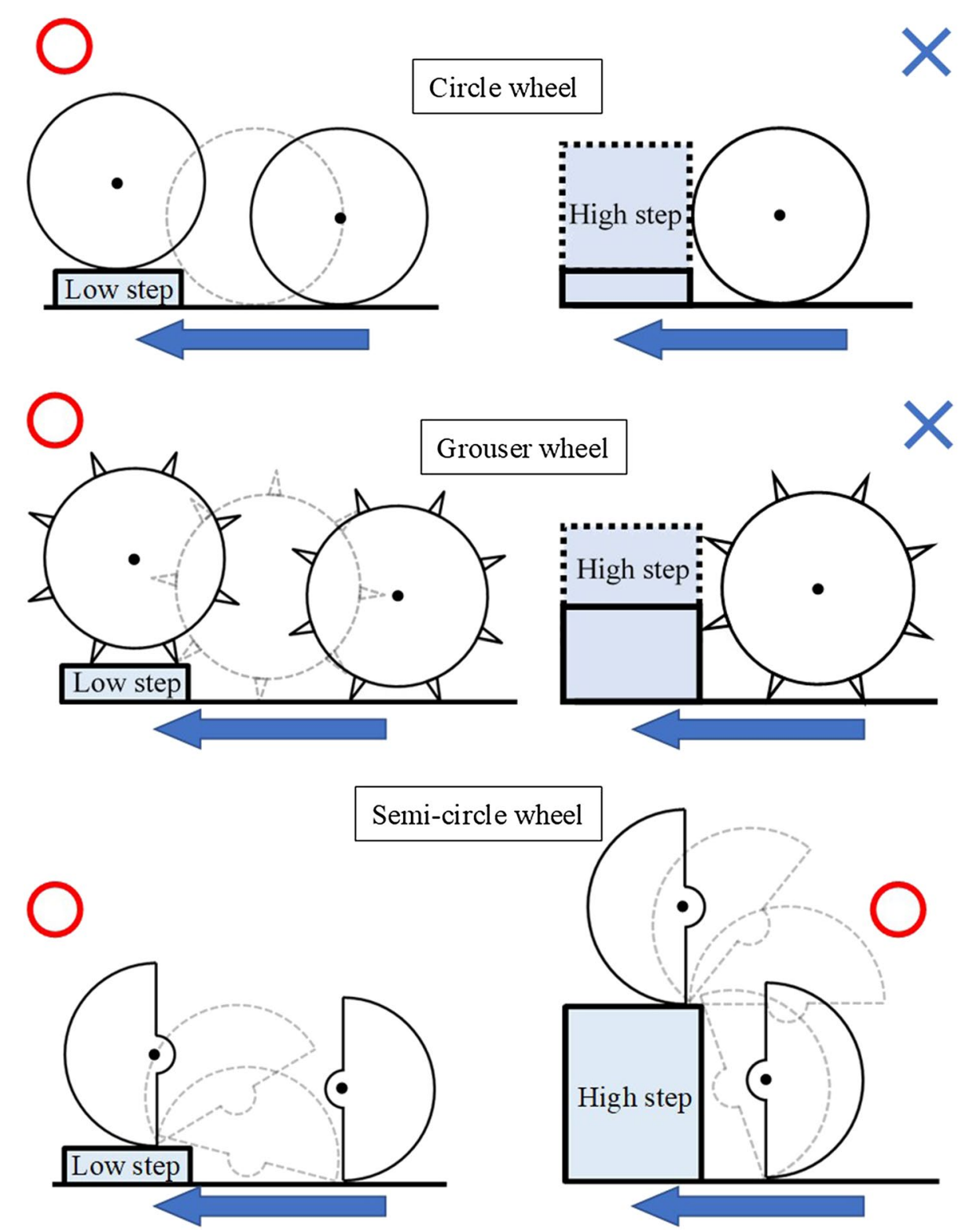

Fig. 1 Comparison of individual wheels

Thus, we introduce crawl gait with a quadruped locomotion to a four-wheeled robot. When crawl gait is performed by a four-legged robot, the center of gravity is always inside the support polygon by the feet on the ground while the main body of the prototype model is kept horizontal. It is therefore possible for the prototype model to move without falling. In a legged robot, its center of gravity portion projected on the ground and support polygon are relatively changed with every moment. However, with our robots, the CoG position and support polygon do not change because the relative position between the wheel's contact point with the ground and the body doesn't change. With only the crawl gait installed in our prototype model, the gait becomes unstable because its center of gravity is not inside its support polygon. We devised a way to move the center of gravity when crawl gait is performed with the prototype model as shown in Fig. 9. The phase changes in the order of (1), (2), (3), (4). The phases of the arc side of the wheels (blue arrows) are respectively shifted by 60 degrees, and the liner side of the wheels (red arrows) rotate at three times the speed of the arc side. This occurs because the liner side is linked to the lifted legs and the arc side, which makes contact with 


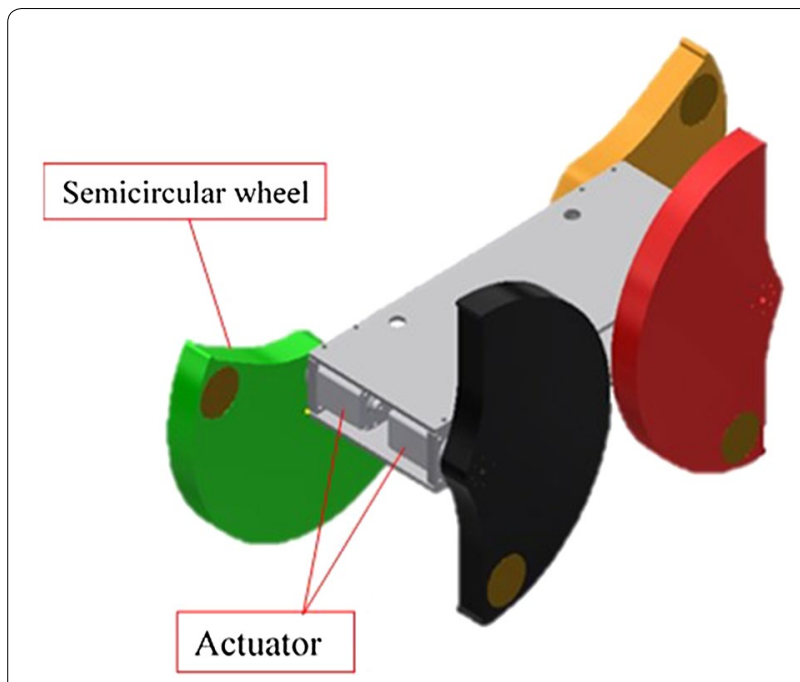

Fig. 2 Basic design

the ground; the legs are rotated with a phase difference of some degrees.

\section{Consideration of moving CoG}

We believed the center of gravity could be moved by rotating wheels with built-in weights at wheel tips (Fig. 5). When the wheel with built-in weight is rotated, the position of the weight changes as shown in Fig. 10. Alternatively, it is possible to change the CoG position moving forward and backward in the sagittal plane. As the wheel changes from $A$ to $B$, the position of the weight changes. It was thought that the center of gravity of the entire prototype model also changed and moved from $C$ to $\mathrm{D}$ and the stable region. It is therefore possible for the prototype to move without falling.

\section{Verification of CoG movement}

We analyzed whether CoG movement in the sagittal plane is possible by having a built-in weight at the tip of all wheels. Equation (1) represents the equation of motion of the centroid coordinates. G is the CoG coordinates; $\mathrm{m}$ is the mass of the weight (127 g); and $\mathrm{Rf}$ is

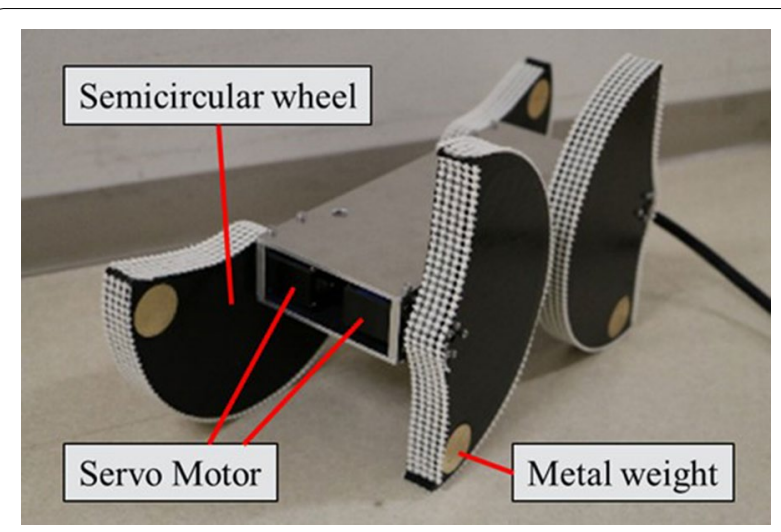

Fig. 4 Semicircular wheels robot

Table 1 Specifications of Semicircular wheel robot

\begin{tabular}{ll}
\hline Length & $420(\mathrm{~mm})$ \\
Width & $149(\mathrm{~mm})$ \\
Height & $200(\mathrm{~mm})$ \\
Weight & $1207(\mathrm{~g})$ \\
Wheel diameter & $200(\mathrm{~mm})$ \\
Metal weight & $127(\mathrm{~g})$ \\
Actuator & KONDO \\
& KAGAKU B3M- \\
& SC-1040-A \\
\hline
\end{tabular}

the horizontal distance from the center of the prototype model to the center of the built-in weight of the right front wheel. Similarly, Rr, Lf, and Lr are the horizontal distances from the center of the prototype model to the center of the built-in weight of the right rear wheel, the left front wheel, and the left rear wheel. Figure 11 shows the state of the prototype model at the crawl gait, letting the direction of travel be X. In the graph of Fig. 12, the vertical axis represents the center of gravity coordinates of the prototype model with respect to the $\mathrm{X}$ axis, and the horizontal axis represents time. Orange areas are states in which one wheel is the liner side and three wheels are in contact with the ground. The green areas are states in
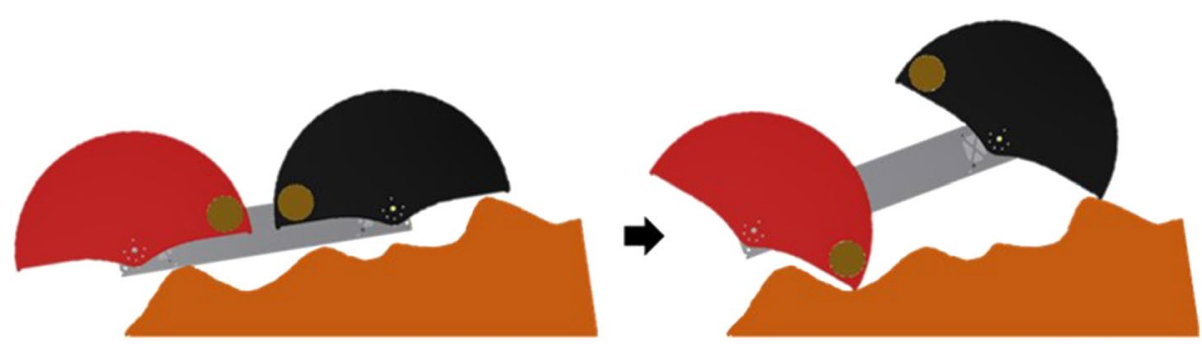

Fig. 3 Phase change of butterfly gait 


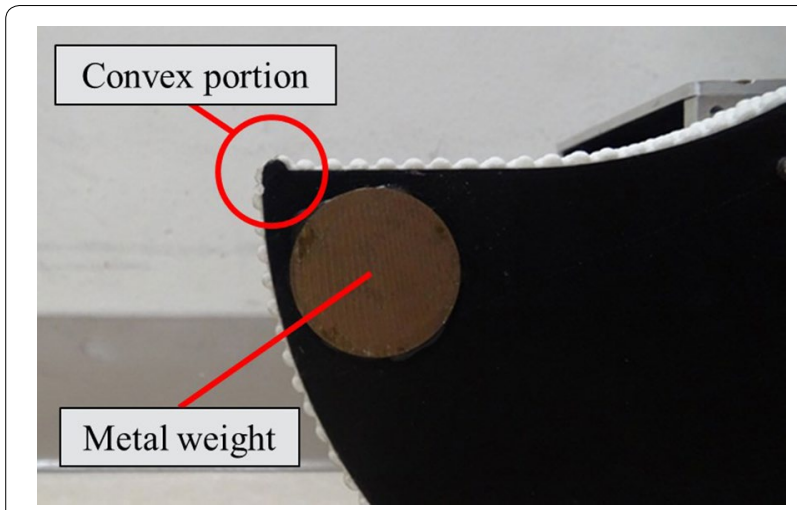

Fig. 5 Wheel tip

which all four wheels are in contact with the ground. In the areas of green and orange, the prototype model is in a state in which it does not overturn. As a result of performing calculations of one circle of crawl gait from the Eq. (1), CoG coordinates were found to be in the stable region in all conditions. Also, it is possible for the prototype to move without falling. At this time, the moving distance of the center of gravity is $\pm 10 \mathrm{~mm}$.

$$
G=\frac{m(R f+R r+L f+L r)}{4 m}
$$

The vertical axis represents the center of gravity coordinates of the prototype model with respect tothe $\mathrm{X}$ axis, and the horizontal axis represents time. Areas of orange are states in which one wheel is alifted leg and three wheels are in contact with the ground.

\section{Motion experiment of crawl gait}

We conducted an experiment to confirm whether the crawl gait, which is the gait of the leg type robot, is possible also with the wheel type robot. This experiment was conducted on flat ground and with an angular velocity of the liner side of 150 degrees and angular velocity at ground contact of 50\% $/$ s. (1) to (6) in Fig. 13 represent the flow until each wheel makes one cycle. Experiments confirmed that the prototype model moved without falling. Moreover, the experiments also confirmed that it is possible to move without an upward and downward motion.

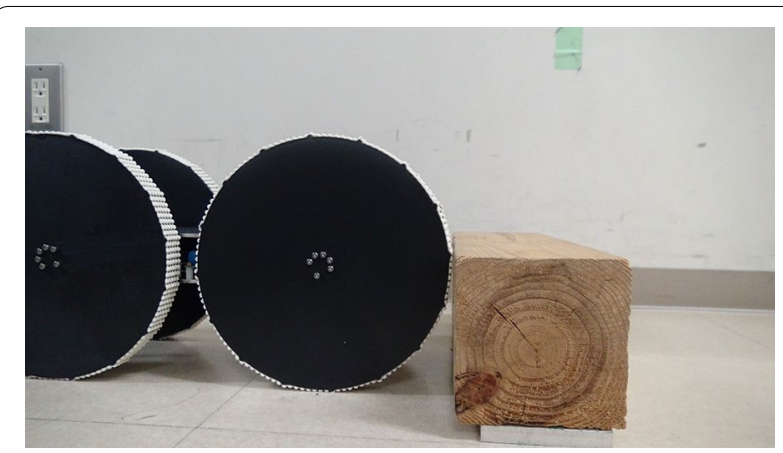

Fig. 7 Grouser gait motion

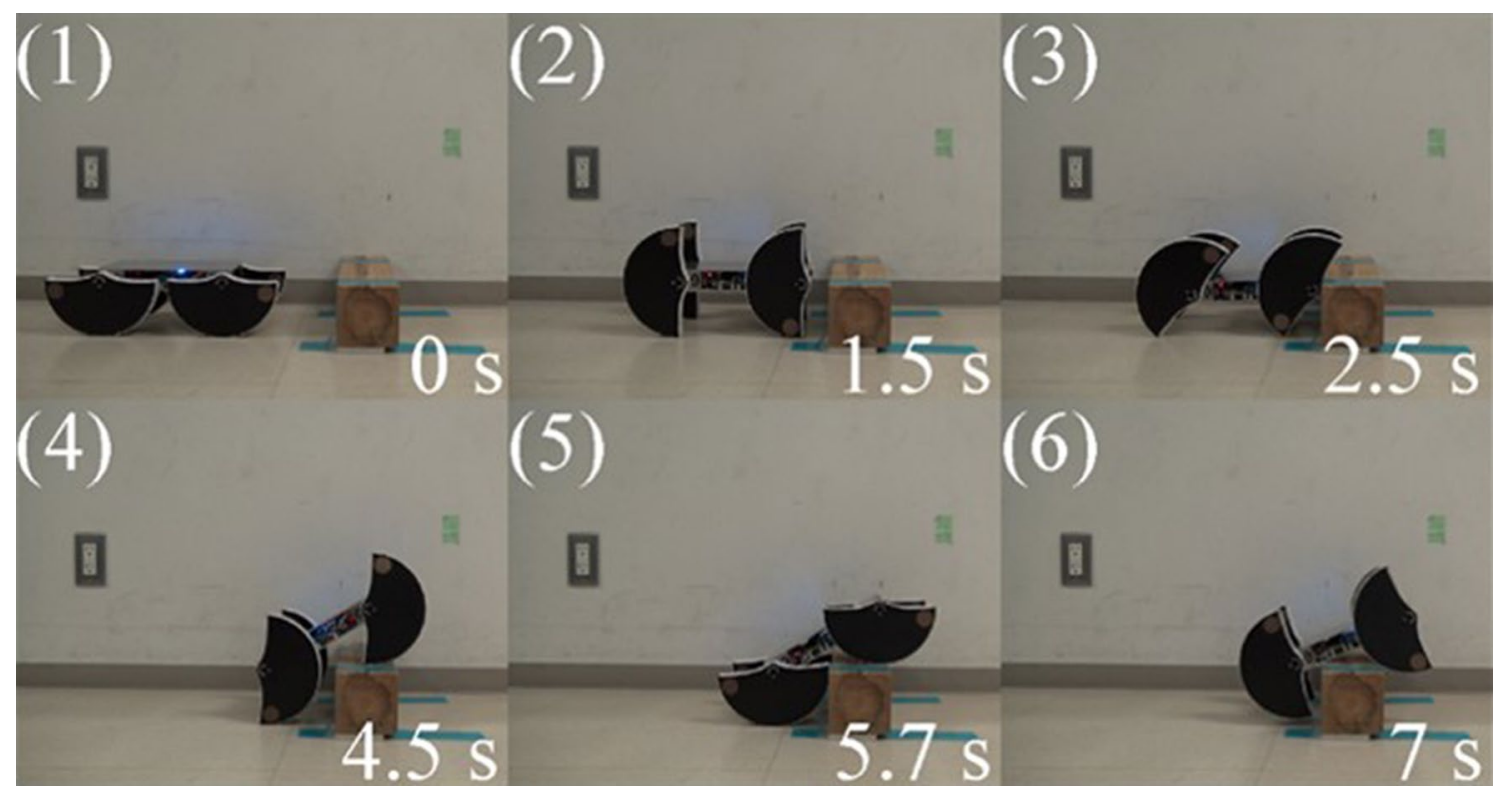

Fig. 6 Butterfly gait motion 


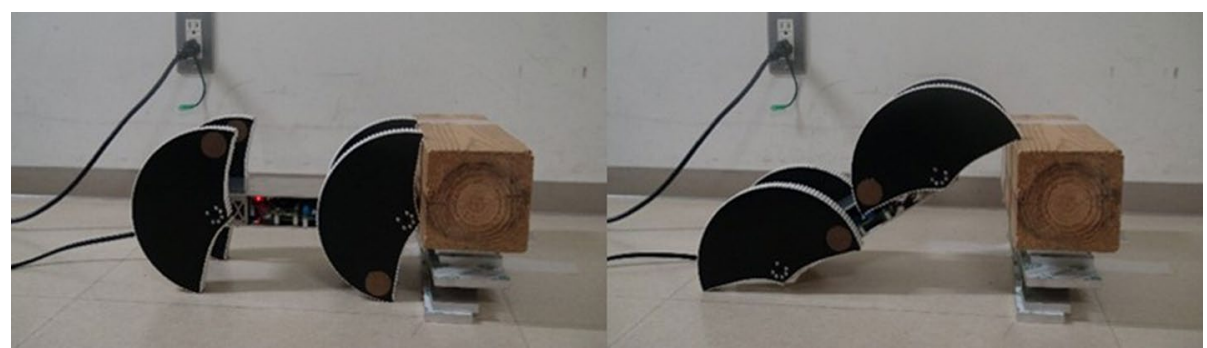

Fig. 8 Step climbing (190 mm)

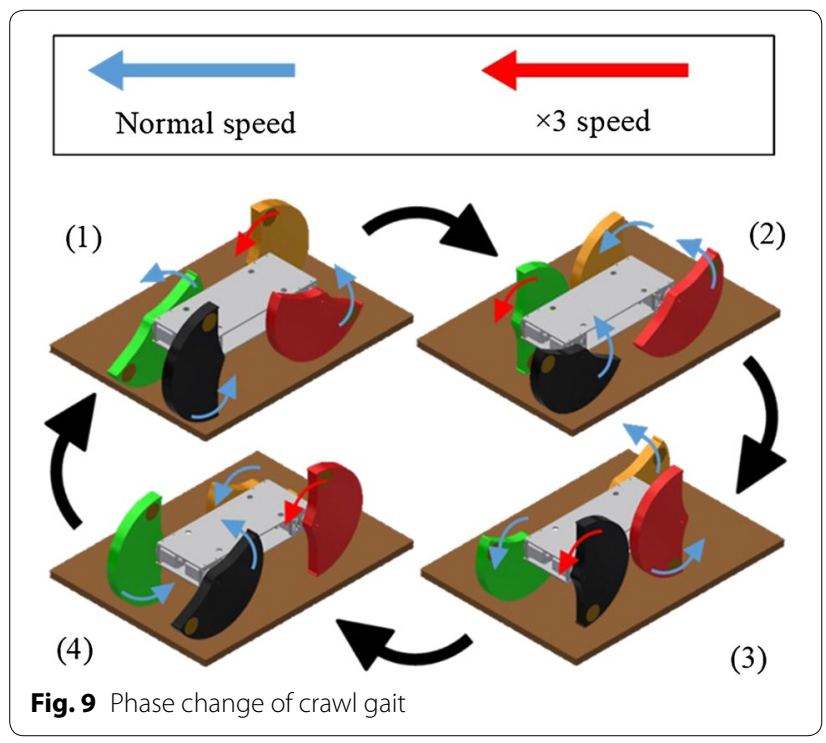

We also verified the extent of upward and downward motion during one circle of the crawl gait without falling. We installed a 6-axis sensor MPU-6050 at the top of the prototype model. We examined model Roll angle and Pitch angle. Figure 14 is the displacement of the Roll angle and Pitch angle with time. It was found that the displacement of the Roll angle and Pitch angle moved only $-1.03^{\circ}$ and $2.79^{\circ}$ at maximum. The experiments confirmed that it is possible to move without upward and downward motion.

\section{Turning motion}

\section{Installation of turning motion}

An independent two-wheeled moving robot can turn by changing the speed of the left and the right wheels. RHex can turn by the speed difference formed between the left and the right legs [13]. If the prototype model moves in the same way by the crawl gait, the difference between the angular velocity of the right and the left wheels collapses the gait. It is necessary to look again at the turning motion. Figure 15 shows the change in the phase of each wheel during turning. The horizontal axis is time and the vertical axis is angular velocity. The blue graph shows the change in the phase of the wheel when it is moving straight forward and the red graph shows the change in the phase of the wheels when turning. In the graph, the slow angular velocity is the contact with the ground; the fast one is the liner side of the semicircular wheel. Time on the ground is increased by making the time of the liner side faster and overlapping with other wheels; it can make a different moving speed of the left and the right wheels. However, because the rotation angles of one cycle are the same on both sides, slow rotation of the wheel can be reproduced. Also, the liner side time gets faster.

\section{Construction of turning motion}

Equations (2), (3) is the formula that forms the basis of the turning motion. $\theta_{R}$ and $\theta_{L}$ are the sum angle of the wheels of both sides in one cycle.

$$
\begin{aligned}
& \boldsymbol{\theta}_{\boldsymbol{R}}=\int_{0}^{\boldsymbol{t}_{\boldsymbol{G}}} \dot{\boldsymbol{\theta}}_{\boldsymbol{R} \boldsymbol{G}} \boldsymbol{d} \boldsymbol{t}+\int_{\boldsymbol{t}_{\boldsymbol{G}}}^{\boldsymbol{t}_{\boldsymbol{S}}} \dot{\boldsymbol{\theta}}_{\boldsymbol{R} \boldsymbol{S}} \boldsymbol{d} \boldsymbol{t} \\
& \theta_{L}=\int_{0}^{t_{G}} \dot{\theta}_{L G} d t+\int_{t_{G}}^{t_{S}} \dot{\theta}_{L S} d t
\end{aligned}
$$

$\dot{\theta}_{R G}$ and $\dot{\theta}_{R S}$ are the angular velocity of the right wheel at ground contact and lift. $\dot{\theta}_{L G}$ and $\dot{\theta}_{L S}$ are the angular velocity of the left wheel at ground contact and lift. $t_{G}$ and $t_{S}$ are times of ground contact and lift. They are the sum of the integral values of the angular velocities at the time on the ground and the time of lift, respectively, which is the sum of the respective angles. Based on this equation, a graph can be represented as shown in Fig. 16. Both wheels can set the speed difference. The wheel on one side can be rotated in the negative direction. Also, when the same angular velocity is reached in the negative direction, it is at pivot turn.

When the absolute value of the sum of the left and the right angles is the same as in Eq. (4), the prototype model can make a turning motion.

$$
\left| \pm \theta_{R}\right|=\left| \pm \theta_{L}\right|
$$



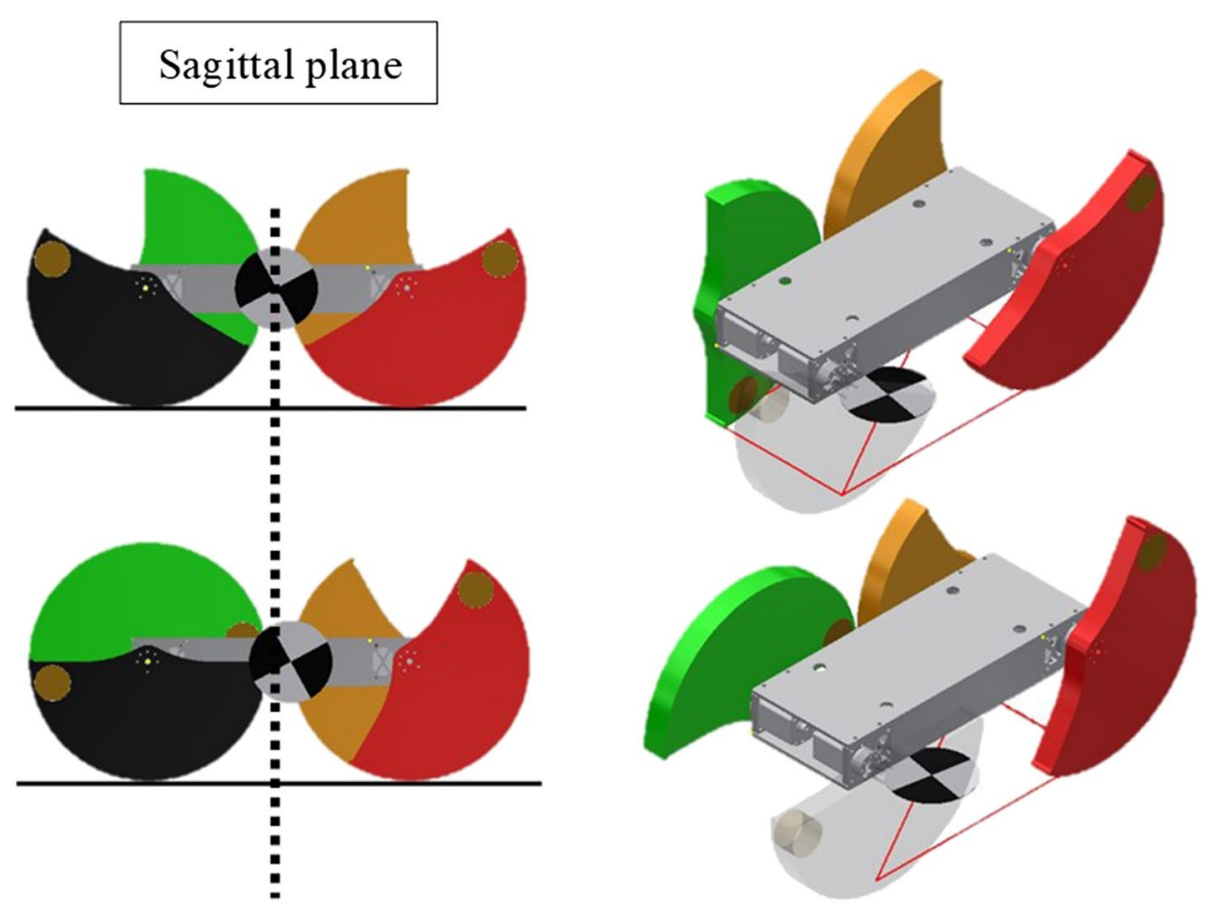

Fig. 10 Stabilization by moving the center of gravity
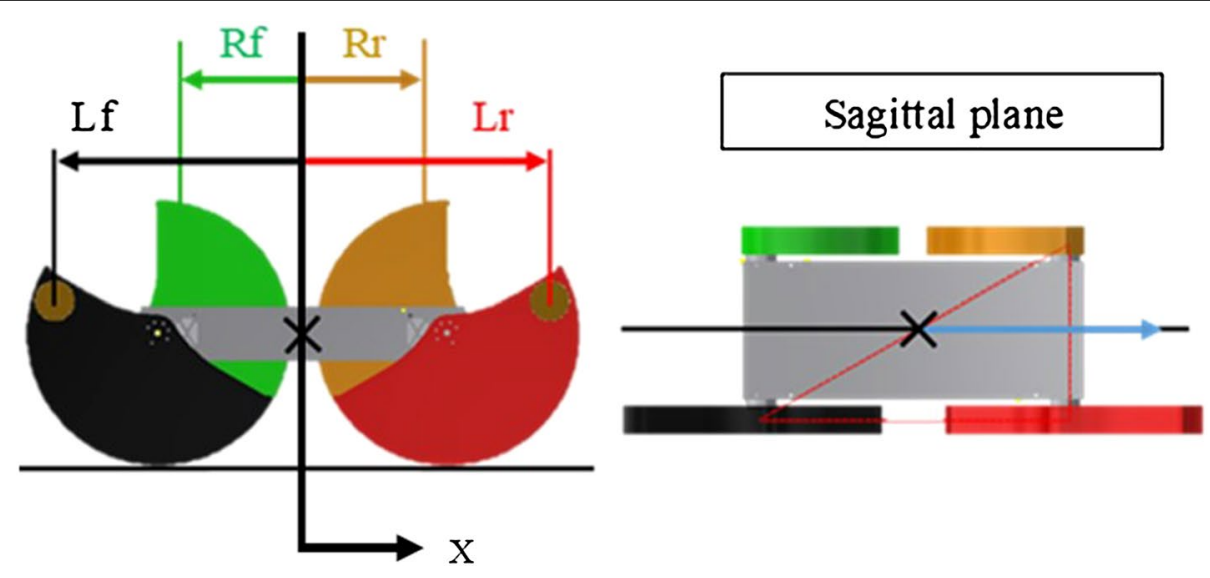

Fig. 11 State of robot at verification

\section{Motion experiment of turning motion}

We conducted the motion experiment of the turning motion on flat ground. On flat ground, the angular velocity at the liner side of the left wheel was $324 \%$, and the angular velocity at ground contact was $5 \%$ s. The angular velocity of the right wheel at the liner side was $19.71^{\circ}$ s and the angular velocity at the moment of ground contact was $6.57^{\circ} / \mathrm{s}$. At this moment the turning radius of the prototype model is $2.89 \mathrm{~m}$. When the speed is making a difference between the left and the right wheels, the upper limit of the rotational speed of the slow wheels is the upper limit of the actuator; the turning speed is rotated at the upper limit of the motor.

To make a turn without falling, the angle at which the wheel makes one cycle must be the same and, as a result, the speed difference between both side wheels cannot be faster, resulting in slower turning. (1) to (6) in Fig. 17 shows the change in the movement until the wheel makes one cycle. The experiment confirmed that the prototype model can turn without falling. 
Coordinate relationship between time and center of gravity at wheel lifted leg (Mass 127[g])

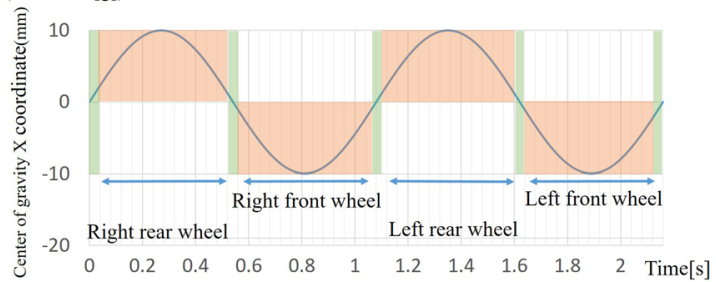

The vertical axis represents the center of gravity coordinates of the prototype model with respect to the $\mathrm{X}$ axis, and the horizontal axis represents time. Areas of orange are states in which one wheel is a lifted leg and three wheels are in contact with the ground.

Fig. 12 A center of gravity coordinate and relations of time

\section{Pivot turn}

Pivot turn is possible when the right and the left wheels have the same angular velocity and reverse direction as the blue and the yellow line in Fig. 16. The basic phase change is the same as the crawl gait, but only one side wheel rotates in the reverse direction. Phase changes in the order of (1), (2), (3), (4) in Fig. 18. The phases of the wheels of the blue arrow are shifted by 30 degrees from each other, and the wheels of the red arrow rotate at three times the speed of the blue arrow.

We analyzed the wheel states in turning motion in the pivot turn. In the graph of Fig. 19, the vertical axis represents the center of gravity coordinates of the prototype model with respect to the $\mathrm{X}$ axis, and the horizontal axis represents time. The green areas are where all wheels are in contact with the ground. In areas of the orange state, one wheel is lifted and three wheels are in contact with the ground. In the areas of green and orange, the prototype model is in a state where it does not overturn. As a result of performing calculations by substituting the weight of the wheel at the time of pivot turning and the center of gravity distance thereof into Eq. (1), as shown in the graph of Fig. 19, we found that the center of gravity coordinates of all the states are in the stable region. Also, pivot turn is possible without the robot falling.

The vertical axis represents the center of gravity coordinates of the prototype modelwith respect to the $X$ axis and the horizontal axis represents time. Orange areas are statesin which one wheel is a lifted leg and three wheels are in contact with the ground.

\section{Motion experiment of pivot turn}

To confirm the possibility of a pivot turn in the prototype, we performed the motion experiment. The experiment was conducted on flat ground, the angular velocity at lifted leg rotation of the left wheel was $-150^{\circ} / \mathrm{s}$, and the angular velocity at ground contact was $-50^{\circ} / \mathrm{s}$. The experiment was conducted with the angular velocity of the lifted leg of the right wheel at $150 \%$ s and the angular velocity at ground contact at $50 \%$ s. (1) to (6) in Fig. 20 are until the prototype model rotates 180 degrees. The

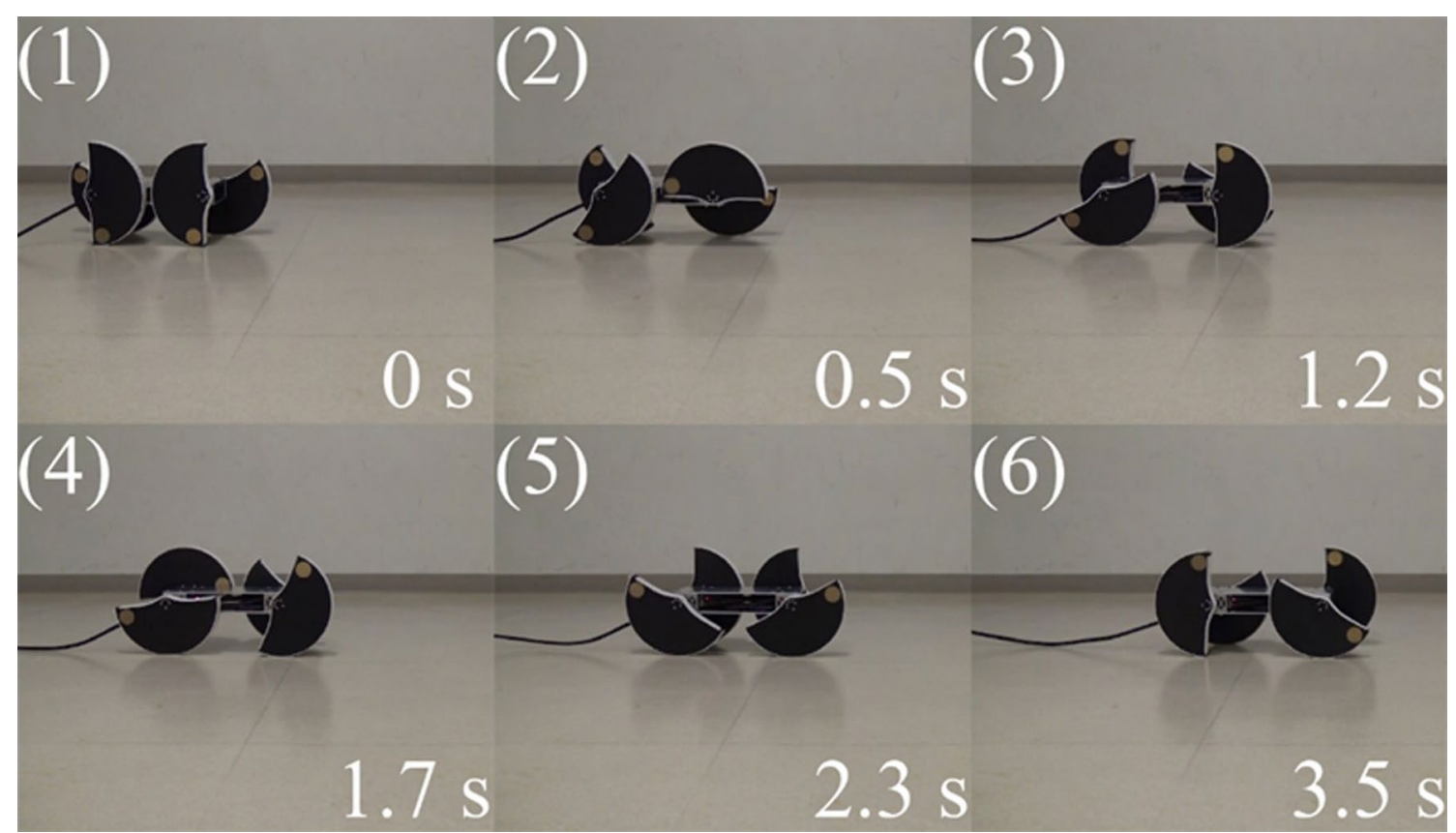

Fig. 13 Crawl gait motion 


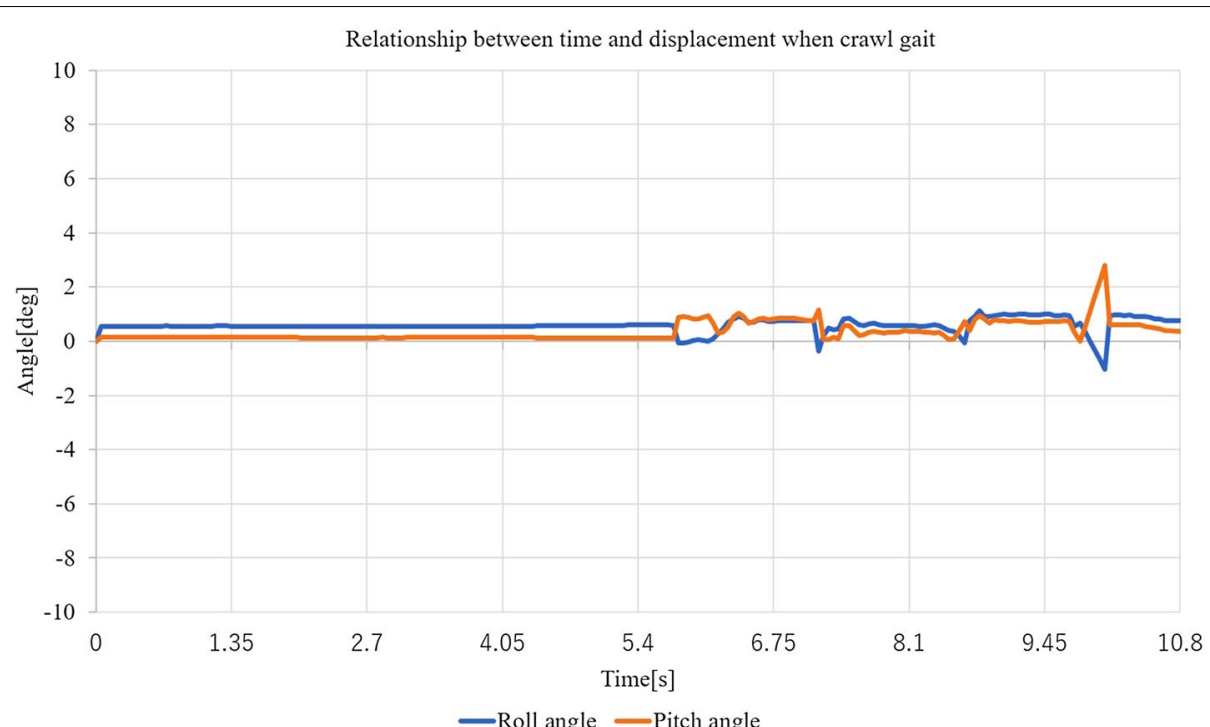

Fig. 14 Relationship between time and displacement
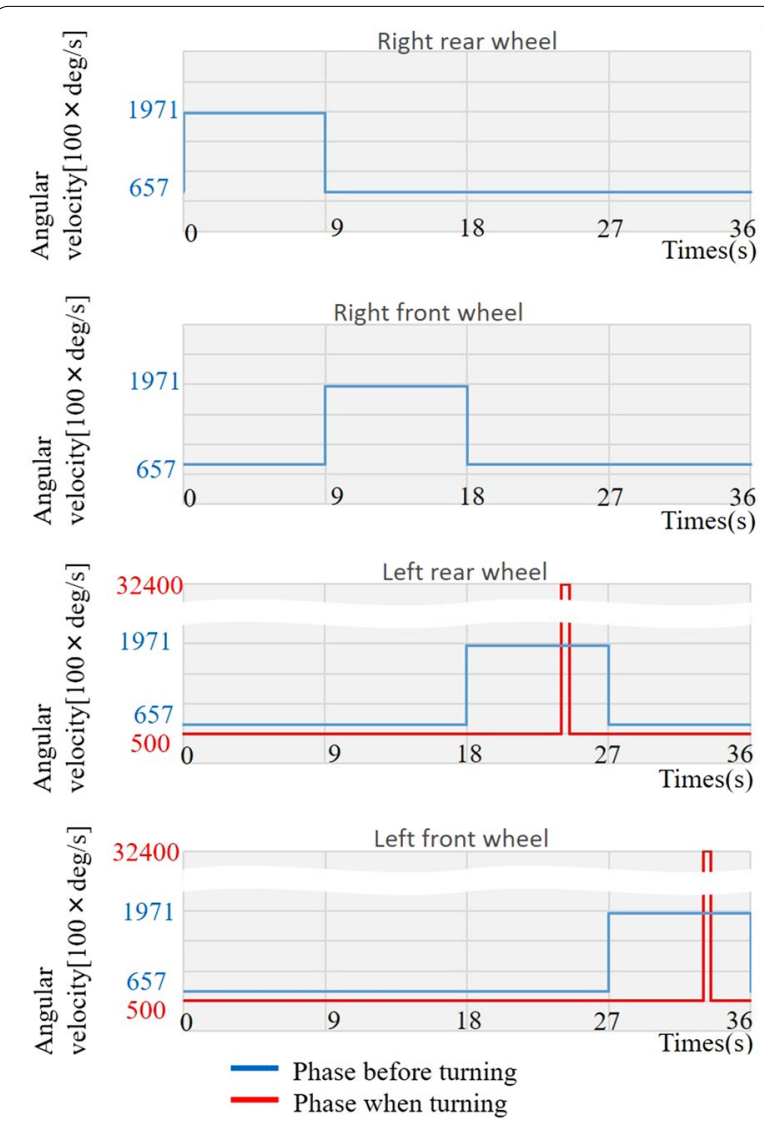

Fig. 15 Phase change of turning motion

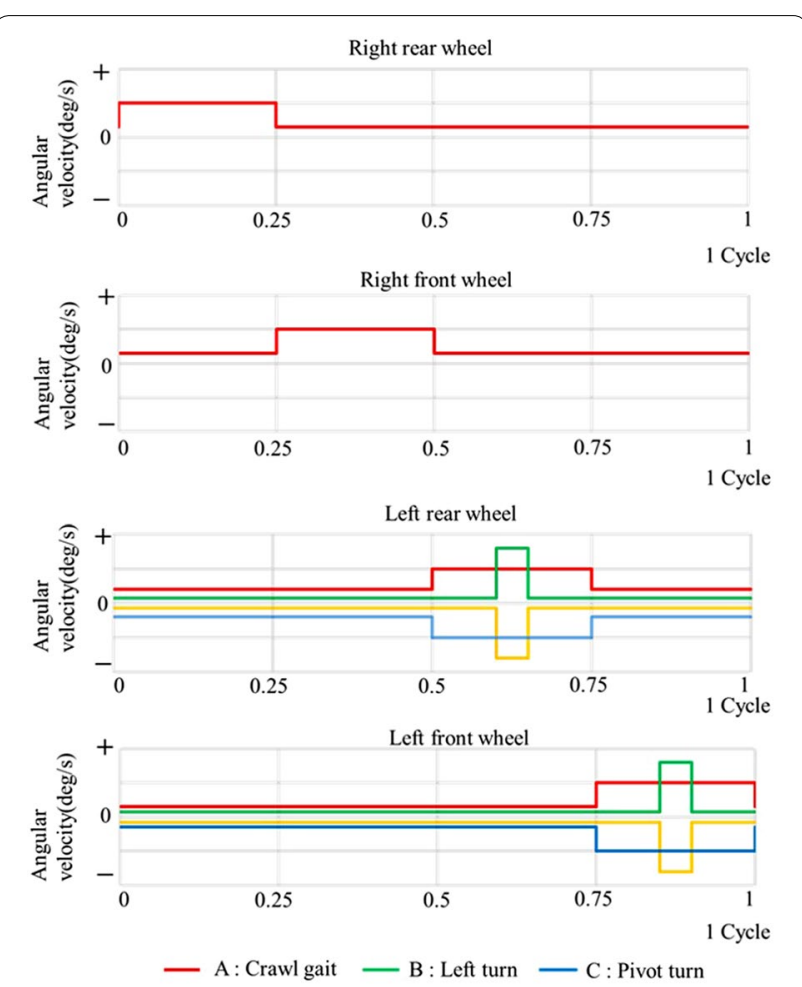

Fig. 16 Continuous phase change from forward movement to pivot turn

experimental results confirmed that the prototype model can pivot turn without falling. 


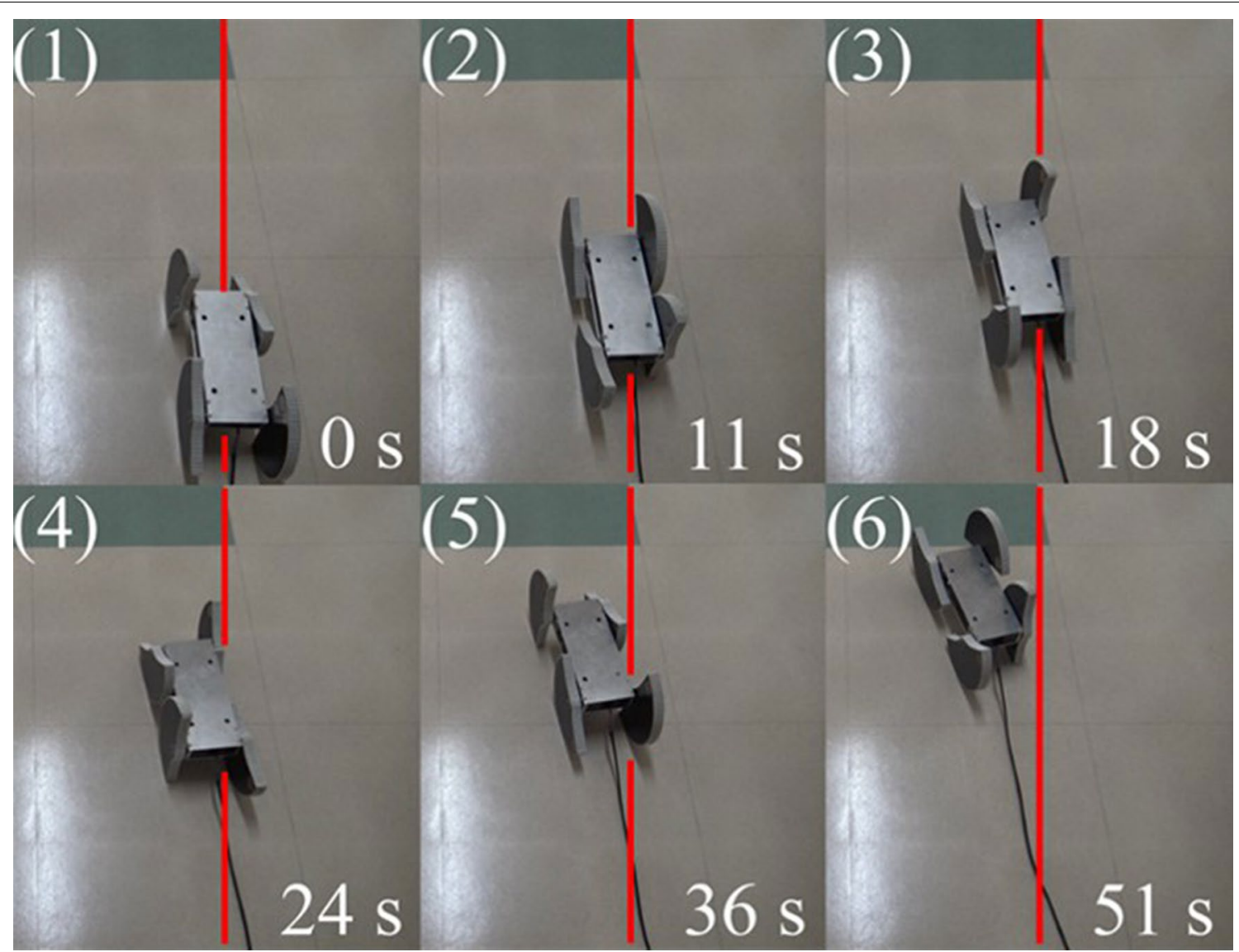

Fig. 17 Turning motion using crawl gait
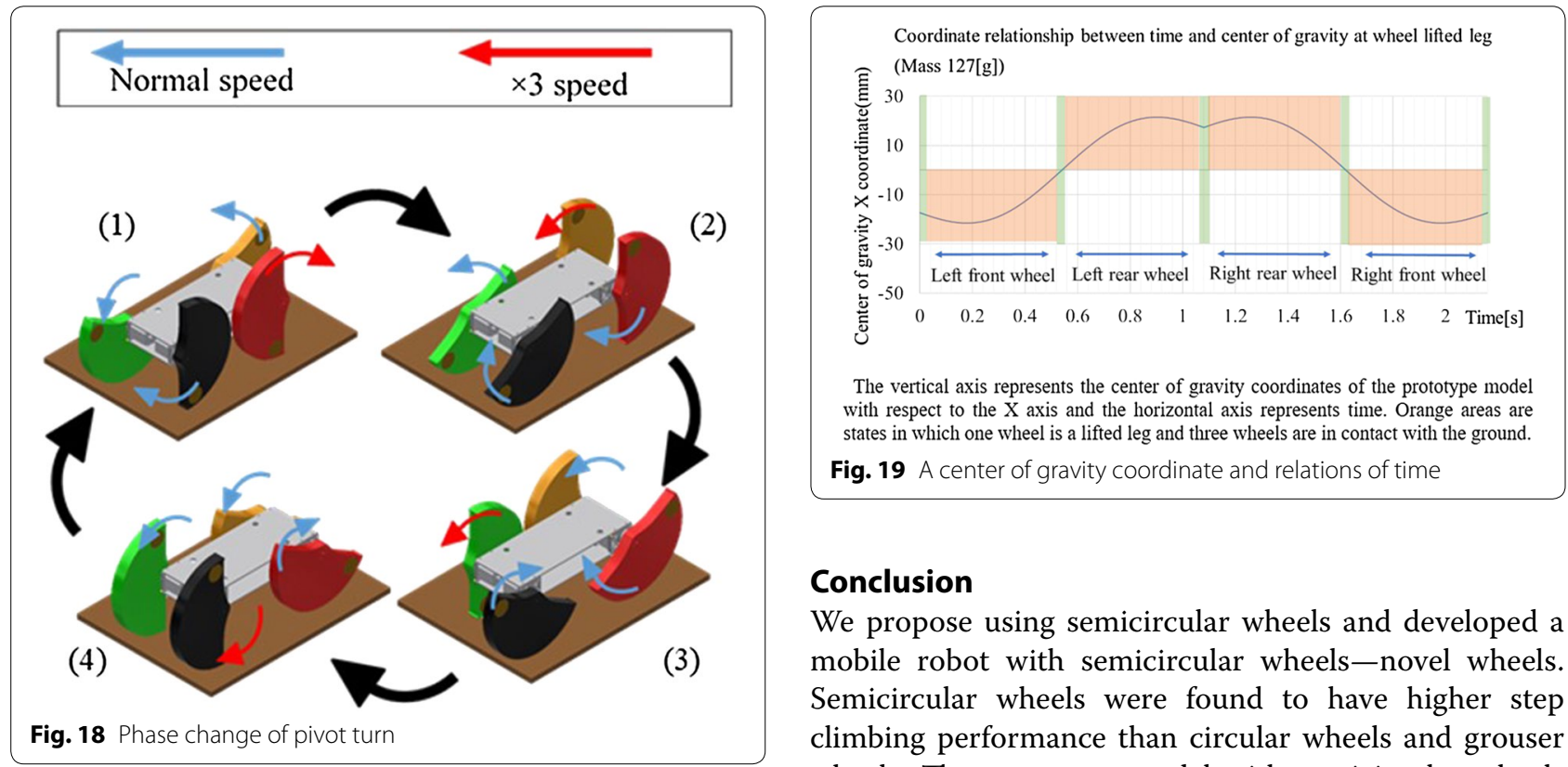

\section{Conclusion}

We propose using semicircular wheels and developed a mobile robot with semicircular wheels-novel wheels. Semicircular wheels were found to have higher step climbing performance than circular wheels and grouser wheels. The prototype model with semicircular wheels showed good performance in climbing high steps using 


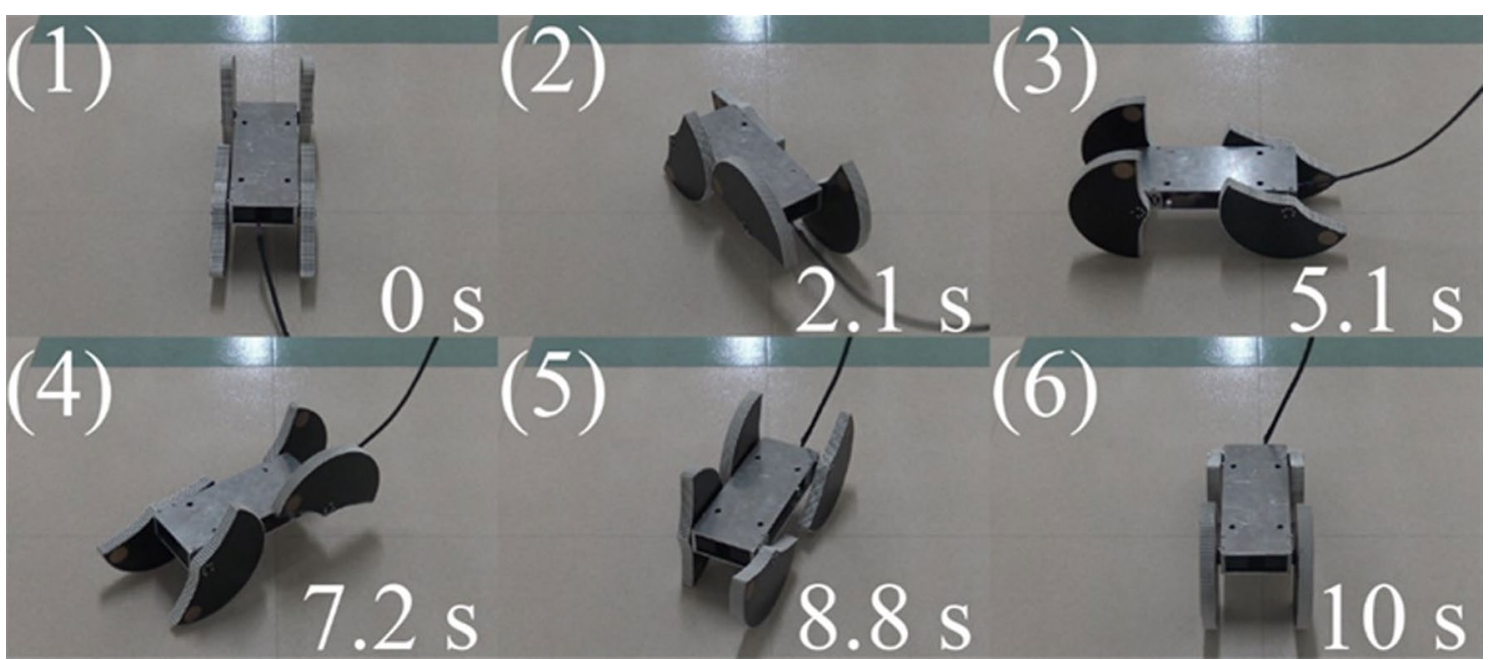

Fig. 20 Pivot turning motion using crawl gait

the butterfly gait. This performance is higher than a robot with circular wheels or grouser wheels. We also confirmed that movement without upward and downward motion is possible on flat ground by the crawl gait. We proposed a new algorithm of turning motion and verified that the prototype model could turn without falling. We also confirmed that it is possible to continuously change from forward turn to pivot turn.

\section{Acknowledgements}

Not applicable.

\section{Authors' contributions}

$\mathrm{KY}$ contributed with concepts and prototype model design, consideration of new locomotion, conducted the experiments, analyzed the data and edited and revised this manuscript. TA contributed with concepts in this manuscript. Both authors read and approved the final manuscript.

\section{Funding}

Not applicable.

\section{Availability of data and materials \\ Not applicable.}

\section{Competing interests}

The authors declare that they have no competing interests.

Received: 15 October 2019 Accepted: 11 August 2020

Published online: 19 August 2020

\section{References}

1. Forsyth RW, Forsyth JP (1967) Amphibious star-wheeled vehicle. U.S. Patent 3348518

2. Yang Y, Qian H, Wu X, Xu G, Xu Y (2012) A novel design of tri-star wheeled mobile robot for high obstacle climbing. In: Proceedings of the IEEE/RS J international conference on intelligent robots and systems. pp 920-925
3. Smith LM, Quinn RD, Johnson KA, Tuck WR (2015) The tri-wheel: a novel wheel-leg mobility concept. In: Proceedings of the IEEE/RSJ international conference on intelligent robots and systems (IROS). pp 4146-4152.

4. Herbert SD, Drenner A, Papanikolopoulos N Loper: a quadruped-hybrid stair climbling robot, In: Proc. IEEE Int. Conf. on robotics and automation (ICRA), 2018. pp 799-804

5. Saranli U, Buehler M, Koditschek DE RHex a simple and highly mobile hexapod robot. Int J Robot Res:616-631

6. Moore EZ, Campbell D, Grimminger F, Buehler M (2002) Reliable stair climbing in the simple hexapod 'RHex'. In: Proceedings of the IEEE international conference on robotics \& automation. pp 2222-2227

7. Wei Z, Song G, Sun H, Qi Q, Liu S, Qiao G (2017) Tep-climbing maneuver for transleg in the wheeled mode. In: Proceedings of the 18th international conference on advanced robotics (ICAR). pp 444-9

8. Wei Z, Song G, Zhang Y, Qiao G (2017) Transleg: a wire-driven leg-wheel robot with a compliant spine. In: Proceedings of the IEEE international conference on information and automation. pp 7-12

9. Landis GA, Jenkins PP (1997) Dust on mars: materials adherence experiment results from mars pathfinder. In: Proceedings of the IEEE photovoltaic specialists conference(PVSC). pp 865-869

10. Choi D, Kim JR, Cho S, Jung S, Kim J ( 2012) Rocker-Pillar: Design of the rough terrain mobile robot platform with caterpillar tracks and rocker bogie mechanism. In: Proceedings of the IEEE/RSJ international conference on intelligent robots and systems. pp 3405-3410

11. Dian S, Liu T, Liang Y, Mengyu, Zhen LW (2011) A novel shrimp roverbased mobile robot for monitoring tunnel power cables. In: Proceedings of the 2011 IEEE international conference on mechatronics and automation. pp 887-892

12. Tsukagoshi H, Kitagawa A, Ito M, Ooe K, Kiryu I, Kochiya T (2008) Bari-bariII: jack-up rescue robot with debris opening function. In: Proceedings of the IEEE international conference on robotics and automation. pp 2209-2210

13. Sun M, Li S, Liu R, Zhang R, Zong G (2009) Tripod gait planning of a rimless-wheeled robot with changeable spokes. In: Proceedings of the 2009 IEEE international conference on robotics and biomimetics. pp $1715-1720$

\section{Publisher's Note}

Springer Nature remains neutral with regard to jurisdictional claims in published maps and institutional affiliations. 